Presented to IAU, GA 23, S 183, Kyoto, August 18-30, 1997.

\title{
Distribution and evolution of quasars
}

S. Nasiri ${ }^{1,2}$ and V. Rezania ${ }^{3}$

1. Dept. of Phys. Zanjan Univ., Zanjan, Iran

2. Center for Theo. Phys. and Math., AEOI, Tehran, Iran

3. Institute for Advanced Studies in Basic Sciences, IASBS, Gava-Zang, Zanjan, Iran.

e-mail: rezania@hotmail.com

In this paper we investigate: a) The evolution of quasars on the basis of gravitational contraction model of proto-galaxies. This is done by studying the number density of quasars given by Veron catalogue [1] in different luminosity classes. The order of classes increase with increasing the luminosity of corresponding quasars. It is shown that the decay of quasars is more sensible to their luminosities as expected by assuming that they are evolved from the contraction of slowly rotating proto-galaxies. The role of the angular momentum in the evolution of galaxies is emphasized by the results obtained from the size-luminosity relation of about 40,000 normal galaxies given by LEDA database [2]. In the model mentioned above the normal galaxies are assumed to be evolved from the contraction of relatively fast rotating proto-galaxies. b) The filamentary structures and voids of the large scale universe by plotting the entire sky map of quasars in the galactic coordinate. This is done by assuming that these objects are at cosmological distances. The result which is plotted in the following figure, seems to show some spatial correlations of quasars distribution. The region of missing quasars is due to the dust clouds in our galaxy which block our view of other quasars. Note the voids and clumpy distribution of quasars looking like filaments.

\section{References:}

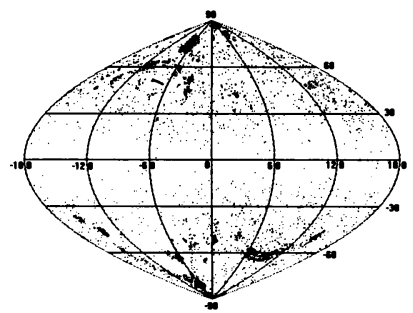

1. Veron, M. P. and Veron, P., A catalogue of quasars and active nuclei, 5th edition, Scientific report, European Southern Observatory, No. 10, 1991.

2. Paturel, G., Bottinelli, L., Di Nella, H., Durand, N., Garnier, R., Gouguenheim, L., Marthinet, M. C., petit, C., Rousseau, J., Therreau, G., Vauglin, L., Lyon-Meudon Extragalctic Database (LEDA), For 100,000 galaxies, Observatoire de Lyon, 1996. 